\title{
Pancreatic metastasis of uterine cervix cancer mimicking a primary cancer
}

\author{
Sho Ogata ${ }^{1 *}$, Akifumi Kimura ${ }^{2}$, Koji Kameda ${ }^{1}$, Hidenori Sasa ${ }^{3}$ and Junji Yamamoto ${ }^{2}$ \\ ${ }^{1}$ Department of Pathology and Laboratory Medicine, National Defense Medical College, Tokorozawa, Japan \\ ${ }^{2}$ Department of Surgery, National Defense Medical College, Tokorozawa, Japan \\ ${ }^{3}$ Department of Gynecology, National Defense Medical College, Tokorozawa, Japan
}

\begin{abstract}
Pancreatic metastasis is rare. We present the case of pancreas metastasis from a uterine invasive cervix cancer, manifested by obstructive jaundice. Bile duct or pancreatic cancer was diagnosed from the laboratory data and imaging studies. During the subsequent pancreaticoduodenectomy, uterine cancer with peritoneal dissemination was found synchronously. The resected specimen contained a whitish tumor located in the pancreas, ampulla, and lower bile duct mimicking infiltrating-type primary cancer. Disseminating metastasis seemed most likely as the route of pancreatic metastasis from the uterus. Tumors located in the pancreas, even those that clinically mimic primary cancers, should be considered as possible secondary tumors.
\end{abstract}

\section{Introduction}

The majority of cancers occurring in the pancreas are of primary ductal origin, and secondary tumors of the pancreas are rare [1-3]. In the past, secondary tumors of the pancreas were usually found in the autopsy. The most common origins include lung, breast, skin (melanoma), stomach, colorectum, kidney, and ovary or, in surgical series, lung, kidney, breast, colorectum, and skin (melanoma) [1]. However, a pancreatic metastasis of uterine cancer is very rare [2, 3]. Indeed, there were none among 103 (reported from Japan [3]) or 81 (reported from the Unites States [2]) cases in autopsy series with secondary tumors of the pancreas. In surgical series, only five cases of a pancreatic secondary tumor arising from cervical cancer have been reported [2,4-7]. Here, we present a Japanese case with a pancreatic metastasis of an invasive cervical cancer that was manifested by obstructive jaundice. A pancreas head tumor was clinically diagnosed from laboratory data and from imaging studies. However, the pancreatic tumor was concluded to be metastatic from her uterine invasive cervix cancer. We describe the lesions' features and discuss the route of metastasis. Further, we emphasize that in such cases the tumor should be ruled out or ruled in as a secondary, although unfortunately few surgeons have so far had the opportunity for such diagnosis.

\section{Clinical summary}

A 78-year-old Japanese woman with a past history of cerebellar infarction complained of appetite loss and general fatigue. She was referred to the National Defense Medical College Hospital (Saitama, Japan), because of jaundice and dilatation of the common bile duct (revealed in an imaging study at a local hospital). Laboratory data revealed elevations in serum bilirubin (direct-bilirubin predominant), aspartate transaminases, alanine transaminases, alkaline phosphatase, $\bigotimes$-glutaryl transpeptidase, and carbohydrate antigen 19-9, while the serum values of carcinoembryonic antigen and Span-1were within normal limits. Serum squamous cell carcinoma (SCC) antigen was not evaluated. Imaging studies revealed luminal stricture and wall irregularity in the inferior bile duct, with dilatation of both the upstream bile duct and the main pancreatic duct (Figure 1). Although repeated bile cytology failed to confirm malignancy, a pancreaticoduodenectomy was planned with a pre-operative diagnosis of pancreatic or bile duct cancer without distant metastases. However, during the operation a small nodule was found within the pouch of Douglas. Unexpectedly, intraoperative pathologic examination revealed it to be a metastatic deposit of SCC. Pelvic examination revealed a cauliflower-like tumor of the uterine cervix, and histology confirmed it to be SCC, papillary variant. We considered the patient to have both a biliary or pancreatic cancer and stage IIIa cervical cancer, and the pancreaticoduodenectomy was completed. She received best-supportive care for a stage IVb cervical cancer. She died 10 months after the operation. An autopsy was not performed.

\section{Pathological findings}

In the resected specimen, accompanying the dilatation of the bile and pancreatic ducts, a whitish tumor, $53 \times 27 \times 12 \mathrm{~mm}$, was located in the periampullary region with involvement of pancreas and the lower bile duct (Figure 2A). The irregular contour of the tumor mimicked the infiltrating type of primary pancreatic cancer. Histologically, the tumor was composed of solid and trabecular nests of tumor cells contacting each other by intercellular bridges, features compatible with SCC (Figure 2B). At the center of some tumor nests, degeneration was observed, although keratinization of tumor cells was indistinct (Figure 2C). Tumor nests spread along the boundary between duodenal wall

Correspondence to: Sho Ogata, Department of Pathology and Laboratory Medicine, National Defense Medical College, 3-2 Namiki, Tokorozawa, Saitama, 359-8513 Japan, Tel: +81-4-2995-1505; fax: +81-4-2996-5192; E-mail: sogata@ ndmc.ac.jp

Key words: pancreas metastasis; cervix cancer; squamous cell carcinoma

Received: April 06, 2018; Accepted: April 20, 2018; Published: April 23, 2018 


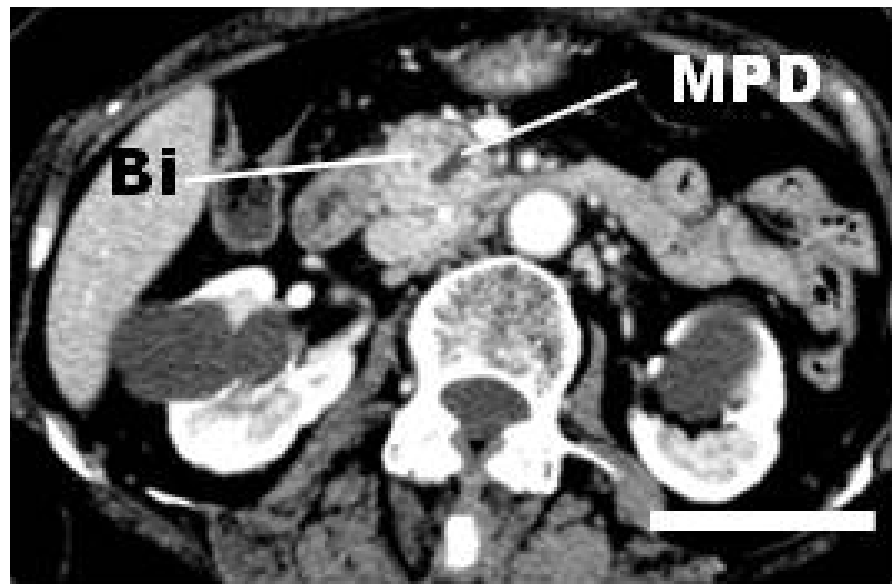

Figure 1. Abdominal computed tomography revealed obstruction of the inferior bile duct (Bi). Dilatation of the upstream main pancreatic duct (MPD) also seen. Scale indicated $3 \mathrm{~cm}$

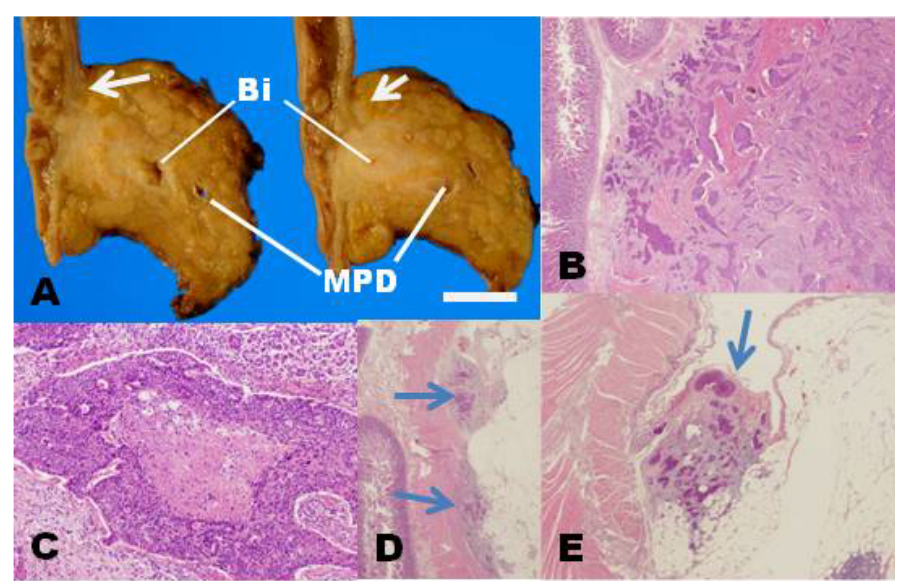

Figure 2. Gross and histologic features of the pancreas head tumor

A. The tumor involved bile duct (Bi), main pancreatic duct (MPD), and duodenum. It mimicked a primary pancreatic cancer with its irregular contour. Note also extension along the boundary between the duodenum and pancreas. Indicated scale, $1 \mathrm{~cm}$. B, C. The tumor was composed of tumor cell nests with the features of squamous cell carcinoma (hematoxylin-eosin). D. Cancer cell nests (arrows) extended along the boundary between the duodenum and pancreas (hematoxylin-eosin). E. Cancer cell nests (arrow) were observed on the peritoneal surface continuous with the boundary between the duodenum and pancreas (hematoxylin-eosin)

and pancreatic parenchyma (Figure 2D), and some were exposed at the serosal surface (Figure 2E). Metastatic deposits were also observed within a few peripancreatic lymph nodes. The tumor was considered a metastatic deposit from her cervical cancer on the basis of the presence of cervical cancer and the lack of glandular differentiation within the pancreatic head tumor.

\section{Discussion}

In surgical series of secondary tumors of the pancreas, such tumors tend to appear symptomatically and are clinically suspected of being primary cancers, especially when tumor is found solitary [1]. In the present case, the obstructive jaundice due to the bile-duct involvement of the tumor led to us finding it. However, the patient did not complain of any gynecologic symptom. Thus, the tumor was mistakenly diagnosed preoperatively as a primary cancer. In a patient with a primary pancreatic or bile duct cancer accompanied by stage III uterine cervical cancer, a pancreaticoduodenectomy might be appropriate, because the 5-year-survival rate for stage III uterine cervical cancer is about $40 \%$, better than for pancreatic or bile duct cancer $[1,8]$. In fact, however, our patient had a stage IVb uterine cancer, for which chemoradiotherapy might be selected as treatment. Even if a given pancreatic tumor displays typical clinical and imaging features, it should be ruled out or ruled in as a secondary by careful examination. Moreover, assessment of the lesion, including intraoperative histologic and/or cytologic examination, might be helpful to determine which therapeutic plan might be appropriate for the patient's disease status.

Secondary tumors of the pancreas are considered to occur via various routes: 1) direct extension, 2) lymphatic, 3) vascular, and/or 4) disseminated routes. In the present case, direct extension was denied, and only peripancreatic lymph nodes displayed involvement of tumor cells. Peritoneal seeding of cancer cells was observed intraoperatively, and tumor-cell nests were characteristically located along the line between the duodenum and pancreas. This might suggest a route of cancer propagation from the peritoneal surface. Although the vascular route could not be completely denied, it seemed reasonable to conclude that metastasis to the pancreas occurred via peritoneal dissemination.

\section{Conflict of interests}

None declared.

\section{Reference}

1. Hruban RH, Pitman MB, Klimstra DS (2007) Tumors of the pancreas. Silverberg SG, ed. Atlas of tumor pathology, 4th series. Washington, DC: American Registry of Pathology.

2. Adsay NV, Andea A, Basturk O, Kilinic N, Nasaar H, et al. (2004) Secondary tumors of the pancreas: an analysis of a surgical and autopsy database and review of the literature. Virchows Arch 444: 527-535. [Crossref]

3. Nakamura E, Shimizu M, Itoh T, Manabe T (2001) Secondary tumors of the pancreas clinicopathological study of 103 autopsy cases of Japanese patients. Pathol Int 51: 686690. [Crossref]

4. Wastell C (1966) A solitary secondary deposit in the pancreas from a carcinoma of the cervix. Postgrad Med J 42: 59-61. [Crossref]

5. Ogawa H, Tsujie M, Miyamoto A, Yasui M, Ikenaga M, et al. (2011) Isolated pancreatic metastasis from uterine cervical cancer: a case report. Pancreas 40: 797-798. [Crossref]

6. Kuwatani M, Kawakami H, Asaka M, Marukawa K, Matsuno Y, et al. (2008) Pancreatic metastasis from small cell carcinoma of the uterine cervix demonstrated by endoscopic ultrasonography-guided fine needle aspiration. Diagn Cytopathol 36: 840-842. [Crossref]

7. Hirose Y, Matsushita T, Yamamoto H (2002) A case of pancreatic tumor metastasizing from uterine cancer. Nihon Rinsho Geka Gakkai Zasshi 65: 1283-1287.

8. Kurman RJ, Ronnett BM, Sherman ME, Wilkinson EJ (2010) Tumors of the cervix, vagina, and vulva. Silverberg SG, ed. Atlas of tumor pathology. 4th series. Washington, DC: American Registry of Pathology.

Copyright: (C2018 Ogata S. This is an open-access article distributed under the terms of the Creative Commons Attribution License, which permits unrestricted use, distribution, and reproduction in any medium, provided the original author and source are credited. 